\title{
Long-term disease control during TAS-102 therapy in the VI line treatment in a 73-year- -old mCRC patient. A case report
}

Piotr Skaliji, ${ }^{1,}$ Piotr Tokajuk, ${ }^{1,2}$, Stefan Jelski ${ }^{3}$, Marek Z. Wojtukiewicz, 2

'Department of Oncology, Medical University of Bialystok

${ }^{2}$ Clinical Oncology Ward with Daytime Chemotherapy unit, Bialostockie Oncology Center

${ }^{3}$ Department of Radiology, Bialostockie Oncology Center

Correspondence:

Marek Z. Wojtukiewicz Department of Oncology, Medical University of Bialystok 15-027 Białystok, ul. Ogrodowa 12

Received: 10.03.2020 Accepted: 26.03.2020

DOI: 10.24292/01.OR. 120260320 Copyright (C) Medical Education. All rights reserved.

\section{ABSTRACT}

A case of 73-year-old patient with metastatic colorectal cancer who underwent trifluridine/tipiracil therapy within the VI line was presented. Treatment with trifluridine/tipiracil allowed to achieve stabilization of the existing cancer for nearly 7 months.

Key words: metastatic colorectal cancer, TAS-102, long-term response 


\section{INTRODUCTION}

Over the past two decades, the prognosis for patients with metastatic colorectal cancer (mCRC) has improved significantly. Thanks to the use of new cytostatics and biological drugs in the therapy of patients with $\mathrm{mCRC}$, the median overall survival (mOS) of patients has significantly increased and currently exceeds 30 months. In the case of progression after standard treatment including fluoropyrimidine derivatives, oxaliplatin, irinotecan, anti-vascular endothelial growth factor (VEGF) antibodies and anti-epidermal growth factor receptor antibodies, some patients remain in good condition. The therapeutic option in these patients is TAS-102, an oral fluoropyrimidine derivative, which is a combination of two active ingredients: trifluridine and tipiracil hydrochloride. Therapy with this drug is relatively well tolerated and can also be used in older and intensively over-treated patients.

The following is a case of a 73-year-old mCRC patient treated with trifluridine/tipiracil as part of systemic IV line treatment.

\section{CASE PRESENTATION}

The patient is a 73-year-old man diagnosed with rectal cancer in July 2014. About $6 \mathrm{~cm}$ behind the sphincter, the primary lesion was accompanied by numerous pelvic and abdominal metastatic lymph nodes described in PET-CT, which was considered as a M1 feature. Due to the above, the patient was relinquished from preoperative radiotherapy and anterior amputation of the rectum was performed. Histopathological postoperative report (pT3) revealed the features of angioinvasion and cancer blood clots in vessels. In September 2014, palliative chemotherapy was introduced according to the FOLFIRI program. Doses of cytostatics were reduced after the first course due to massive G3 diarrhea, but no other complications were observed. In December 2014, the progression of the disease was observed in the form of enlargement of metastatic lymph nodes. FOLFOX chemotherapy with bevacizumab was initiated, which the patient continued until November 2015 (XVII courses). The treatment was further complicated by single episodes of G1 neutropenia as well as worsening polyneuropathy and proteinuria leading to the termination of current therapy despite the stabilization of the disease in imaging tests. The patient remained under observation until September 2016, when the disease progressed. Genetic testing of the primary tumor tissue material did not reveal mutations in the RAS genes, which enabled the start of anti-EGFR therapy with panitumumab. The targeted therapy was well tolerated by the patient with complications observed were acne-like skin lesions in G1/G2 only. Treatment was terminated in May 2017 due to dis- ease progression in the abdominal lymph nodes. Due to the persistent small extent of the disease as well as good general condition of the patient, monotherapy with capecitabine was set up.

The first imaging control after 3 months revealed further progression of the disease resulting in an attempt to return to FOLFIRI chemotherapy at reduced doses as at the first line of treatment. The above treatment was continuously administered until March 2018. In May 2018, hepatic metastatic lesion was found. It was effectively treated with thermoablation, after which the patient was observed. In October 2018, CT scan revealed disease progression in the liver and lymph nodes, metronomic chemotherapy (capecitabine $3 \times 500 \mathrm{mg}+$ cyclophosphamide 50 mg) was started. In March 2019, metronomic therapy was terminated due to diarrhea and disease progression, including the appearance of new metastases in the liver, adrenal gland and a single metastasis to the skeletal system ( $\mathrm{L} 4$ vertebra). In April 2019, TAS-102 was received as part of the manufacturer's drug donation.

The patient remained in good general condition, except for the slight neuropathy remaining after oxaliplatin treatment. Trifluridine/tipiracil treatment was introduced at the appropriate dose. After the second course, the patient presented G3 neutropenia, G2 anemia and G2 diarrhea. Once these side effects were no longer present, treatment was continued by $1 / 3$ dose reduction. Control imaging performed at that point revealed partial regression of the metastatic change in the adrenal gland, a reduction of metastases in other parenchymal organs, which indicated the disease stabilization and an enlargement of metastasis in bones. The patient underwent palliative radiotherapy for the L4 vertebra $(1 \times 8 \mathrm{~Gy})$. At the same time, TAS-102 chemotherapy was continued at a reduced dose with no clinically significant complications. Up until now (November 2019), the patient still continues treatment, remaining in good general condition without any symptoms of the disease.

\section{DISCUSSION}

In the case described above, a steady development of the disease lasting 4 years was observed in the patient suffering from $\mathrm{mCRC}$. Throughout this time, the patient underwent therapy with all systemic therapies available in Poland, including anti-VEGF and anti-EGFR antibodies. Patients representing this group seem to be good candidates for trifluridine/tipiracil therapy in the context of prior biological therapy. When comparing the results of the main studies evaluating the effectiveness of TAS-102 in patients suffering from mCRC (TERRA and RECOURSE studies) who 
had previously received anti-VEGF and anti-EGFR therapy (in RAS WT patients), the clinical benefit of TAS-102 appears to be greater [1, 2]. The patient's general condition has a significant impact on the effectiveness of TAS-102, as demonstrated by European analyses $[3,4]$. The patient presented above was in good general condition (according to ECOG 1) when starting trifluridine/ tipiracil treatment. Importantly, the patient's good general condition continued throughout the entire therapy period, while the results from registration studies suggested the median time necessary to indicate deterioration of fitness to be 5.7 months. The 7-month treatment period without the disease progression is much longer than in the registration study, currently reaching a value close to the median OS achieved in this study. Complications observed in the patient concerned mainly hematological, which is consistent with the toxicity profile of TAS-102. In the phase III of clinical trials neutropenia of grade $\geq 3$ occurred in more than $1 / 3$ of patients receiving TAS 102. Moreover, both anemia and diarrhea were more frequently observed during treatment within the study drug when compared to the control group. The reduction of the drug dose enabled the continuation of treatment while maintaining therapeutic efficacy.

\section{CONCLUSION}

To sum up, during the therapy with trifluridine/tipiracil therapy, stabilization of the existing neoplastic disease was achieved in the $\mathrm{mCRC}$ patient described by us for a period of about 7 months. It should be emphasized that it was possible to maintain a fairly good quality of life for the patient while maintaining a satisfactory performance state according to the ECOG scale.

\section{References}

1. Mayer RJ, Van Cutsem E, Falcone A et al. Randomized trial of TAS-102 for refractory metastatic colorectal cancer. N Engl J Med. 2015; 372(20): 1909-19.

2. Xu J, Kim TW, Shen L et al. Results of a randomized, double-blind, placebo-controlled, Phase III trial of trifluridine/tipiracil (TAS-102) monotherapy in Asian patients With previously treated metastatic colorectal cancer: the TERRA study. J Clin Oncol. 2018; 36(4): 350-8.

3. Kwakman JJM, Vink G, Vestjens JH et al. Feasibility and effectiveness of trifluridine/tipiracil in metastatic colorectal cancer: real-life data from The Netherlands. Int J Clin Oncol. 2018; 23: 482-9.

4. Cremolini C, Rossini D, Martinelli E et al. Trifluridine/tipiracil (TAS-102) in refractory metastatic colorectal cancer: a multicenter register in the frame of the Italian compassionate use program. Oncologist. 2018; 23: 1178-87.

Authors' contributions: Piotr Skalii: project of the paper, preparation of the manuscript; Piotr Tokajuk: preparation of the manuscript; Stefan Jelski: preparation of the manuscript; Marek Z. Wojtukiewicz: project of the paper, preparation of the manuscript.

Financial support: None.

Ethics:

The authors had full access to the data and take full responsibility for its integrity. 